\title{
Characteristics and influencing factors of soil microbial communities in different subtropical forest stands in Jinyun Mountain, Southwest China
}

\section{Xiaonan An}

Beijing Forestry University https://orcid.org/0000-0002-3494-454X

Yunqi Wang ( $\square$ wangyunqi@bjfu.edu.cn )

Beijing Forestry University

Jialiang Zhang

Beijing Forestry University

\section{Research}

Keywords: Soil microbial community, Phospholipid fatty acid, Forest soil, Subtropical forests

Posted Date: September 3rd, 2021

DOl: https://doi.org/10.21203/rs.3.rs-858115/v1

License: (c) (i) This work is licensed under a Creative Commons Attribution 4.0 International License.

Read Full License 


\section{Abstract}

\section{Background}

An insight into the soil microbial functions and spatial distribution of soil resources is an important basis for evaluating and managing plant growth in subtropical forests. Soil samples were collected from five forest stands in Jinyun Mountain Natural Reserve (JMNR) in Chongqing located at the Three Gorges Reservoir area: Gordonia acuminata evergreen broad-leaved forest (GAEBF), Cunninghamia lanceolata forest (CLF), Phyllostachys pubescens forest (PPF), coniferous and broad-leaved mixed forest (CBLMF) dominated by Pinus massoniana and Gordonia acuminata (PM\&GA), and the CBLMF dominated by Pinus massoniana and Symplocos setchuensis (PM\&SS). Combined with phospholipid fatty acid (PLFA) analysis and Sherlock microbial identification system (MIS), the structure of soil microbial communities in different forest stands was investigated.

Results

The results showed that the PLFAs of soil microorganisms under the forest in JMNR have a high diversity. The PLFA dominance values of the five stands were 16:0, 19:0 cyclo $\omega 7 c, 18: 0,15: 0$ iso and 16 :0 10-methyl. Furthermore, soil microorganisms are dominated by Gram-negative bacteria, and the PLFAs content of soil bacteria in different forest stands is higher than that of fungi PLFAs. Regarding the phospholipid fatty acid biomarkers, the two CBLMFs are the highest, followed by CLF and GAEBF and PPF is the least. Moreover, the proportion of microorganisms in the soil of different forest stands varies. Among them, MP\&SS has the highest gram-negative bacteria, gram-positive bacteria, actinomycetes and fungi.

Conclusions

RDA analysis shows that the main influencing factors of PLFAs in the soil of different forest stands are the content of iron oxide, aluminium oxide, organic matter and total nitrogen in the soil, which are considered to be able to reflect the soil nutrient status of JMNR effectively.

\section{Background}

The soil is a reservoir of a vast and diverse microbial population, which is considered a rich source of many types of soil microorganisms that play an essential role in soil decomposition, nutrient cycling, bioremediation, soil organic matter stabilization, and soil aggregate formation (Dangi et al. 2014; Dangi et al. 2018; Dangi et al. 2019). The soil microbial community (SMC) dominates the coupled chemical and biological processes of the Earth's biosphere by virtue of its quantity and metabolic capacity, and its composition and function analysis can effectively monitor the changes of the forest ecosystem to understand the cycle of elements in the soil such as carbon, nitrogen, and phosphorus (Wang et al. 2019). Moreover, microorganisms are sensitive indicators of soil health (Bao et al. 2020), and their community structure and diversity can quickly respond to environmental changes and stress (Alexander and Bryans 
2006; Wei et al. 2004). Currently, biomarker abundance has been used to clarify significant changes in the structure and function of microbial communities under different land-uses or management patterns, e.g., the number of Gram-negative bacteria (G-) and Gram-positive bacteria (G+) will change drastically to adapt to environmental stress conditions such as soil acidification and metal pollution (Barrow et al. 2004; Rousk et al. 2010). Barrow et al. (2004) confirmed that the fungi/bacteria (F/B) ratio could be used to characterize the response of SMCs to different environmental stressors. Furthermore, phospholipid fatty acid (PLFA) is considered a method for qualitatively and quantitatively analyzing the diversity of SMC, which can estimate the microbial biomass and its community structure according to the species specificity of the PLFA composition and content (Zuo et al. 2016).

The activity and function of soil microorganisms are significantly affected by various external factors, such as physical factors (humidity, temperature), biological factors (predation, competition), chemical factors ( $\mathrm{pH}, \mathrm{CEC}$, dissolved nutrients, salinity, organic matter) and Human activities (Herrick and Hefin 2013; Weil and Brady 2017). In terms of forest ecosystems, forest type, altitude, soil physical and chemical properties, and root system characteristics are the most important factors affecting its SMC (Peay et al. 2011; Shen et al. 2013). Furthermore, soil nutrient content is an important factor affecting vegetation growth, and its accumulation, distribution and circulation are affected by various types of forest stands (Xiang et al. 2014). Fierer et al. (2012) found that complex plant species are beneficial to increase the carbon source diversity and microbial habitat in the soil. Plant roots are the key channel connecting plants and soil, absorbing nutrients and water in the soil environment to support plant growth while releasing secretions and generating stimulating effects to affect the activity of microorganisms and change the structure and function of SMC (Xu et al. 2021). Moreover, as important factors that reflect soil properties, iron oxide, aluminium oxide, and cation exchange capacity can affect the availability of microbial nutrients and the function of microbes (Wang et al. 2018; Chen et al. 2020). Furthermore, vegetation types will also affect the diversity of SMC, e.g., Ding et al. (2015) studied SMC in Shennongjia forest district and found that the diversity of SMC in coniferous forests is lower than that of shrubs forests.

Jinyun Mountain Natural Reserve (JMNR) is located in Beibei District, Chongqing, known as "Emei Mountain in Eastern Sichuan" due to its abundant plant types. Nevertheless, relatively few studies have paid attention to the soil microbes of JMNR ( $\mathrm{Li}$ et al. 2012; Zeng and He 2016), and the soil factors and structure of SMC under different forest types are still not well understood. Studies have shown that the surface soil of JMNR is rich in microorganism population, but it is easily affected by the external environment, especially the environmental stress caused by acid rain (Hu et al. 2015; Frostegård et al. 1993). In this case, this study used PLFA analysis and Sherlock Microbial Identification System (MIS) to investigate the surface soils of typical forest stands in JMNR, e.g., Gordonia acuminata (GA) evergreen broad-leaved forest (EBF), Cunninghamia lanceolata forest (CLF), Phyllostachys pubescens forest (PPF), coniferous and broad-leaved mixed forest. Thus, the community structures of fungi, bacteria, actinomycetes were explored, and the actinomycetes' influence on the SMC of subtropical forests was investigated. In addition, a comparative analysis of the number of soil microorganisms in different forest stands of JMNR was carried out to clarify the factors affecting the structure and distribution of SMC. The 
research results are beneficial for an in-depth understanding of forest types' impact on soil fertility and the relationship between SMC and forest productivity, providing a reference for the realization of the ecological benefits of forest stands and the management and protection of forest resources.

\section{Materials And Methods}

\subsection{Overview of the study area}

JMNR is located in Beibei District, Chongqing ( $\left.106^{\circ} 17^{\prime} 43^{\prime \prime}-106^{\circ} 24^{\prime} 50^{\prime \prime} \mathrm{E}, 29^{\circ} 41^{\prime} 08^{\prime \prime}-29^{\circ} 52^{\prime} 03^{\prime \prime} \mathrm{N}\right)$, with an area of $7,600 \mathrm{hm}^{2}$, which is one of the best-preserved mid-subtropical EBF plots in the Yangtze River Basin in China (Fig. 1). The forests in this site experiences a typical northern subtropical humid monsoon climate with hot and rainy summers and cold and dry winters. JMNR is characterized by foggy weather, with an average annual temperature of $13.6^{\circ} \mathrm{C}$, average annual precipitation of $1611.8 \mathrm{~mm}$, average annual relative humidity of more than $85 \%$, average annual evaporation of $700-800 \mathrm{~mm}$, and average annual sunshine duration of 1293h. Furthermore, Jinyun Mountain has an average elevation of $951.5 \mathrm{~m}$, and the terrain is flat, with a relative elevation difference of $600 \mathrm{~m}$. The soil of JMNR is mainly composed of paddy soil and acid yellow soil ( $\mathrm{pH}$ 4.0-4.5) formed by weathering of the parent materials such as Upper Triassic Xujiahe Formation sandstone, carbonaceous shale, and argillaceous sandstone. Moreover, JMNR has diverse vegetation types, with a forest coverage rate of $96.6 \%$ and a relatively stable forest ecosystem. Specifically, the study area is mainly distributed with large areas of EBF, CBLMF, evergreen broad-leaved shrubs and bamboo forest communities.

\subsection{Collection of samples}

In early August 2019, the soils under five forest stands, namely GA, CLF, PPF, PM\&GA, and PM\&SS, were selected as the research objects. Four quadrats were selected for each stand type, and the area of each quadrat is $12 \times 12 \mathrm{~m}$. Three sampling points were selected for each quadrat. After that, the soil depth of each sampling point was $0-20 \mathrm{~cm}$. Then, the soil was mixed according to the quarter method, and $1 \mathrm{~kg}$ of mixed soil samples were taken as one sample, and 20 samples were obtained in total. The soil sample was enclosed with a pre-cleaned polyethylene terephthalate (PET) bag and placed in an incubator to bring it back to the laboratory. Subsequently, $50 \mathrm{~g}$ of soil samples were taken and stored in a refrigerator at $-20{ }^{\circ} \mathrm{C}$ for subsequent experimental analysis, and the remaining soil samples were prepared for physical and chemical properties analysis. The details of the sampling points are shown in Table 1 . 
Table 1

Basic overview of sampling points.

\begin{tabular}{|llllll|}
\hline $\begin{array}{l}\text { Forest sample } \\
\text { plot }\end{array}$ & $\begin{array}{l}\text { Altitude } \\
(\mathbf{m})\end{array}$ & Aspect & $\begin{array}{l}\text { Canopy density } \\
(\%)\end{array}$ & Origin & Main tree species \\
\hline GA- EBF & $846 \sim 896$ & Northwest & 0.7 & Natural & GA, Neolitsea aurata \\
\hline CLF & $842 \sim 887$ & Northwest & 0.8 & Natural & CL, PM \\
\hline PPF & $745 \sim 790$ & Northwest & 0.75 & Natural & PP \\
\hline PM\&GA & $654 \sim 687$ & Northwest & 0.9 & Natural & $\begin{array}{l}\text { PM, GA, Adinandra } \\
\text { millettii }\end{array}$ \\
\hline PM\&SS & $698 \sim 765$ & Northwest & 0.9 & Natural & PM, SS \\
\hline
\end{tabular}

\subsection{Determination of soil factors}

The physical and chemical analysis process of the soil samples is as follows. After being air-dried naturally at room temperature, the soil samples were ground and sieved $(2 \mathrm{~mm}, 1 \mathrm{~mm}, 0.25 \mathrm{~mm})$. Then, the potassium dichromate external heating method was used to determine the soil organic matter, the automatic chemical analyzer was used to determine the total soil nitrogen, the hydrometer method was used to carry out soil structure analysis, and the ammonium acetate exchange method was used to determine the cation exchange capacities. Furthermore, the free iron oxides (Fed) and free aluminium oxides (Ald) were extracted by the DCB method, and amorphous iron oxides (Feo) and amorphous aluminium oxides (Alo) were extracted by ammonium oxalate and measured by ICP. Moreover, the soil available phosphorus is measured by colourimetry, and the soil pH is measured by the potentiometric method. See Table 2 for the soil's basic physical and chemical properties obtained after repeated measurements of various indicators for each sample three times.

Ditto for selection of soil root quadrat. Samples were collected using a custom soil drill (radius $6.4 \mathrm{~cm}$, height $20 \mathrm{~cm}$ ), and soil was collected once for each site. A total of 20 root samples were collected from 5 stands. 
Table 2

Soil physical and chemical properties of different forest stand1.

\begin{tabular}{|c|c|c|c|c|c|c|c|}
\hline $\begin{array}{l}\text { Forest sample } \\
\text { plot }\end{array}$ & $\mathrm{pH}$ & $\begin{array}{l}\text { SOC } \\
/\left(g \cdot \mathrm{kg}^{-1}\right)\end{array}$ & $\begin{array}{l}\mathrm{TN} \\
/\left(\mathrm{g} \cdot \mathrm{kg}^{-1}\right)\end{array}$ & $\begin{array}{l}\text { AP } \\
/\left(\mathrm{mg} \mathrm{kg}^{-1}\right)\end{array}$ & $\begin{array}{l}\text { Clay } \\
/(\%)\end{array}$ & $\begin{array}{l}\text { Sand } \\
/(\%)\end{array}$ & $\begin{array}{l}\text { Silt } \\
/(\%)\end{array}$ \\
\hline GA- EBF & $\begin{array}{l}4.1 \pm \\
0.2 \mathrm{a}\end{array}$ & $\begin{array}{l}58.6 \pm \\
16.5 a\end{array}$ & $\begin{array}{l}3.2 \pm \\
1.3 a\end{array}$ & $2.9 \pm 2.5 a$ & $\begin{array}{l}15.6 \pm \\
1.2 \mathrm{abc}\end{array}$ & $\begin{array}{l}69.0 \pm \\
2.6 \mathrm{c}\end{array}$ & $\begin{array}{l}15.4 \pm \\
2.9 a\end{array}$ \\
\hline CLF & $\begin{array}{l}4.0 \pm \\
0.2 \mathrm{a}\end{array}$ & $\begin{array}{l}65.6 \pm \\
9.6 a\end{array}$ & $\begin{array}{l}3.5 \pm \\
0.5 a\end{array}$ & $\begin{array}{l}4.7 \pm \\
1.1 \mathrm{ab}\end{array}$ & $18.7 \pm 0.6 c$ & $\begin{array}{l}52.6 \pm \\
1.5 a\end{array}$ & $\begin{array}{l}28.8 \pm \\
0.9 b\end{array}$ \\
\hline PPF & $\begin{array}{l}4.5 \pm \\
0.1 \mathrm{~b}\end{array}$ & $\begin{array}{l}44.0 \pm \\
12.1 \mathrm{a}\end{array}$ & $\begin{array}{l}2.2 \pm \\
0.6 \mathrm{a}\end{array}$ & $\begin{array}{l}10.7 \pm \\
6.0 \mathrm{~b}\end{array}$ & $\begin{array}{l}14.8 \pm \\
0.8 \mathrm{ab}\end{array}$ & $\begin{array}{l}59.4 \pm \\
2.2 \mathrm{ab}\end{array}$ & $\begin{array}{l}25.8 \pm \\
1.4 b\end{array}$ \\
\hline PM\&GA & $\begin{array}{l}4.1 \pm \\
0.2 \mathrm{a}\end{array}$ & $\begin{array}{l}57.2 \pm \\
10.8 a\end{array}$ & $\begin{array}{l}3.1 \pm \\
1.0 \mathrm{a}\end{array}$ & $3.7 \pm 2.0 \mathrm{a}$ & $13.0 \pm 0.8 a$ & $\begin{array}{l}58.2 \pm \\
2.6 a b\end{array}$ & $\begin{array}{l}28.7 \pm \\
1.8 b\end{array}$ \\
\hline PM\&SS & $\begin{array}{l}4.0 \pm \\
0.2 \mathrm{a}\end{array}$ & $\begin{array}{l}43.8 \pm \\
11.7 a\end{array}$ & $\begin{array}{l}2.9 \pm \\
0.9 a\end{array}$ & $3.8 \pm 1.2 \mathrm{a}$ & $\begin{array}{l}17.2 \pm \\
2.7 \mathrm{bc}\end{array}$ & $\begin{array}{l}50.2 \pm \\
11.7 a\end{array}$ & $\begin{array}{l}32.6 \pm \\
9.1 \mathrm{~b}\end{array}$ \\
\hline $\begin{array}{l}\text { Forest sample } \\
\text { plot }\end{array}$ & $\begin{array}{l}\text { Fed2 } \\
/\left(\mathrm{g} \cdot \mathrm{kg}^{-1}\right)\end{array}$ & $\begin{array}{l}\text { Ald2 } \\
/\left(\mathrm{g} \cdot \mathrm{kg}^{-1}\right)\end{array}$ & $\begin{array}{l}\text { Feo2 } \\
/\left(\mathrm{g} \cdot \mathrm{kg}^{-1}\right)\end{array}$ & $\begin{array}{l}\text { Alo2 } \\
/\left(\mathrm{g}^{\mathrm{kg}}{ }^{-1}\right)\end{array}$ & $\begin{array}{l}\text { CEC } \\
/\left(\mathrm{cmol}^{\prime} \mathrm{kg}^{-1}\right)\end{array}$ & & \\
\hline GA- EBF & $\begin{array}{l}16.0 \pm \\
3.0 \mathrm{a}\end{array}$ & $\begin{array}{l}13.2 \pm \\
4.6 \mathrm{a}\end{array}$ & $\begin{array}{l}3.1 \pm \\
0.4 a\end{array}$ & $3.0 \pm 0.7 a$ & $16.4 \pm 3.9 b$ & & \\
\hline CLF & $\begin{array}{l}17.8 \pm \\
3.2 \mathrm{a}\end{array}$ & $\begin{array}{l}15.5 \pm \\
3.8 a\end{array}$ & $\begin{array}{l}4.0 \pm \\
0.4 a\end{array}$ & $3.8 \pm 0.5 a$ & $\begin{array}{l}15.4 \pm \\
1.2 a b\end{array}$ & & \\
\hline PPF & $\begin{array}{l}12.8 \pm \\
4.6 a\end{array}$ & $\begin{array}{l}8.7 \pm \\
4.0 \mathrm{a}\end{array}$ & $\begin{array}{l}3.1 \pm \\
1.1 \mathrm{a}\end{array}$ & $2.7 \pm 1.2 \mathrm{a}$ & $9.9 \pm 3.6 a$ & & \\
\hline PM\&GA & $\begin{array}{l}13.4 \pm \\
2.1 \mathrm{a}\end{array}$ & $\begin{array}{l}10.9 \pm \\
2.4 a\end{array}$ & $\begin{array}{l}3.3 \pm \\
0.6 \mathrm{a}\end{array}$ & $2.6 \pm 0.7 a$ & $\begin{array}{l}11.4 \pm \\
2.3 a b\end{array}$ & & \\
\hline PM\&SS & $\begin{array}{l}15.4 \pm \\
5.3 a\end{array}$ & $\begin{array}{l}8.6 \pm \\
1.9 a\end{array}$ & $\begin{array}{l}2.5 \pm \\
1.2 \mathrm{a}\end{array}$ & $2.6 \pm 0.8 a$ & $9.5 \pm 2.8 a$ & & \\
\hline
\end{tabular}

${ }^{1}$ Different lowercase letters indicate the significance of the difference in soil composition for different forest sample plots $(P<0.05)$.

2 Oxalate extraction is denoted by a suffix "o"; DCB extraction denoted by "d".

\subsection{Determination of root indexes}

The root scanner (Epson Expression 12000XL, Japan) and Win RHIZO Pro (LA2004, Canada) are used to measure root indexes such as root length, root surface area, root volume and average diameter (AD). After being dried, the roots are weighed, and the root length density (RLD), root mass density (RMD), the proportions of fine roots $(0.2 \sim 1 \mathrm{~mm})$ and very fine roots $(\leq 0.02 \mathrm{~mm})$ in total root biomass, and specific root length (SRL) are calculated. Each index is also measured repeatedly three times.

\subsection{Extraction and analysis of PLFA}


PLFA analysis is used to determine and name the SMC by the procedure described by Frostegard et al. (1993) The biomarkers were detected by Sherlock Microbial Identification System (MIS) Version 4.5 (MIDI Inc., Newark, DE, USA), with Hydrogen serving as the carrier gas, and Agilent 7890 series gas chromatograph (Agilent Technologies, Santa Clara, CA) equipped with an FID detector as the hardware platform. Fatty acid quantification was characterized by peak area and internal standard curve method, and the content of each fatty acid was quantified $\left(\mathrm{nmol} \cdot \mathrm{g}^{-1}\right)$ by the internal standard (C19:0) in a single sample. The PLFA content is calculated according to the method described by Abaye et al. (Abaye et al. 2005), and the calculation formula is given as follows.

$$
m F=(P F A M E \times C n g, \text { std } \times V) /(P I S T D \times M n g, \text { std } \times W)
$$

Where PFAME and PISTD represent each esterified fatty acid and the peak area of internal standard respectively; $c_{n g, \text { std }}$ is the internal standard concentration $\left(\mathrm{ng} \mathrm{LL}^{-1}\right) ; M_{n g \text {, std }}$ is the molar mass of the internal standard; $V(\mu \mathrm{L})$ represents the solution volume; $W(\mathrm{~g})$ represents the weight of dry soil.

\subsection{Data processing and analysis}

Microsoft Excel 2019 was used for initial data collation, and Statistical Package for the Social Sciences (IBM SPSS 26.0) was used for the analysis of variance (ANOVA, $P<0.05$ ). Thus, the difference among the physical/chemical properties, root characteristics, and microbial PLFA content of different forest stand soils was tested by multiple comparisons. When $p<0.05$, the difference was considered to have statistical significance. In addition, Principal Component Analysis (PCA) and Redundancy Analysis (RDA) were performed on soil environmental factors and SMC structure through CANOC05.0.

\section{Results}

\subsection{Spatial distribution of soil factors}

Table 2 shows that the soil pH of the sample plots under the five forest stands in Jinyun Mountain are all acidic, and the soil pH of GA-EBF, CLF, PPF, PM\&GA, PM\&SS are 4.1, 4.0, 4.5, 4.1, 4.0, respectively. Moreover, the differences in soil pH of GA-EBF, CLF, PM\&GA, PM\&SS were not significant $(P<0.05)$, while the $\mathrm{pH}$ value of PPF (4.5) was higher than that of the other four forest stands with a statistically significant difference $(P>0.05)$. Furthermore, the soils under the five forests have no significant differences in the contents of soils organic carbon (SOC), total nitrogen (TN), Fed and Ald, and Feo and Alo. As far as the content of available phosphorus (AP) is concerned, the performance of different forest stands is $P P F>C L F>P M \& S S>P M \& G A>G A-E B F$, and there are significant differences between GA-EBF and PPF, CLF and PM\&GA, PPF and PM\&SS. In terms of cation exchange capacity (CEC), GA-EBF > CLF > $M \& G A>P P F>P M \& S S$. Among them, the CEC of PPF is significantly different from that of GA-EBF and PM\&SS. 
The soil texture analysis results showed that the five forest stands had significant differences in the proportion of clay, silt and sand. In addition, the proportions of sand in the soil are all the largest for the five forest stands, especially the proportion of sand in GA is as high as 70\%.

\subsection{Differences in root characteristics of forest stands}

Among the five forest stands, the RMD and RLD of PPF are greater than those of the other four stands, and the differences are significant. Specifically, the order of RMD from largest to smallest is PPF > $P M \& G A>G A>C L F>P M \& S S$; in terms of RLD, the performance is PPF > PM\&GA >CLF > GA > PM\&SS (Table 3). Moreover, the SRL of PM\&SS is the largest among the five forest stands, followed by PPF, and the smallest is GA. Among them, PM\&SS is significantly different from the other four stands, PPF is only significantly different from PM\&SS, and there is no significant difference between other stands.

Furthermore, the proportion of very fine roots in total root biomass is significantly different among the five forest stands. The proportions of very fine roots in PPF and PM\&SS are greater than $30 \%$, while only $15 \%$ in CLF. There was no significant difference in the proportion of root AD and fine root in total root biomass in the five forests.

Table 3

Soil root system characteristics of different forests1.

\begin{tabular}{|c|c|c|c|c|c|c|}
\hline $\begin{array}{l}\text { Forest sample } \\
\text { plot }\end{array}$ & $\begin{array}{l}\mathrm{RMD} /\left(\mathrm{kg} \cdot \mathrm{m}^{-}\right. \\
3)\end{array}$ & $\begin{array}{l}\text { RLD } \\
/\left(\mathrm{km} \cdot \mathrm{m}^{-3}\right)\end{array}$ & $\begin{array}{l}\text { SRL } \\
/\left(m \cdot g^{-1}\right)\end{array}$ & $\begin{array}{l}\mathrm{AD} \\
/(\mathrm{mm})\end{array}$ & $\begin{array}{l}0.2-1 \mathrm{~mm} \\
/(\%)\end{array}$ & $\begin{array}{l}0-0.2 \mathrm{~mm} \\
/(\%)\end{array}$ \\
\hline GA- EBF & $2.9 \pm 0.3 a$ & $\begin{array}{l}18.2 \pm \\
3.7 \mathrm{a}\end{array}$ & $6.7 \pm 1.8 \mathrm{a}$ & $\begin{array}{l}0.72 \pm \\
0.15 a\end{array}$ & $\begin{array}{l}64.6 \pm \\
9.3 a\end{array}$ & $\begin{array}{l}17.3 \pm \\
8.7 a b\end{array}$ \\
\hline CLF & $2.8 \pm 0.9 a$ & $\begin{array}{l}20.7 \pm \\
7.9 a\end{array}$ & $7.3 \pm 1.2 \mathrm{a}$ & $\begin{array}{l}0.74 \pm \\
0.11 \mathrm{a}\end{array}$ & $\begin{array}{l}66.4 \pm \\
8.8 \mathrm{a}\end{array}$ & $15.4 \pm 9.2 a$ \\
\hline PPF & $6.6 \pm 2.7 b$ & $\begin{array}{l}33.4 \pm \\
5.4 \mathrm{~b}\end{array}$ & $\begin{array}{l}9.4 \pm \\
0.9 \mathrm{ab}\end{array}$ & $\begin{array}{l}0.66 \pm \\
0.04 a\end{array}$ & $\begin{array}{l}51.1 \pm \\
3.8 \mathrm{a}\end{array}$ & $33.8 \pm 4.6 c$ \\
\hline PM\&GA & $3.3 \pm 0.7 a$ & $\begin{array}{l}21.1 \pm \\
6.6 \mathrm{a}\end{array}$ & $7.2 \pm 1.2 a$ & $\begin{array}{l}0.76 \pm \\
0.04 a\end{array}$ & $63 \pm 11 a$ & $\begin{array}{l}18.1 \pm \\
7.7 \mathrm{ab}\end{array}$ \\
\hline PM\&SS & $1.3 \pm 0.2 a$ & $\begin{array}{l}12.4 \pm \\
1.1 \mathrm{a}\end{array}$ & $\begin{array}{l}10.6 \pm \\
1.5 c\end{array}$ & $\begin{array}{l}0.67 \pm \\
0.04 a\end{array}$ & $\begin{array}{l}51.1 \pm \\
7.3 a\end{array}$ & $\begin{array}{l}30.9 \pm \\
7.5 \mathrm{bc}\end{array}$ \\
\hline \multicolumn{7}{|c|}{$\begin{array}{l}{ }^{1} \text { Different lowercase letters indicate the significance of the differences in soil root characteristics for } \\
\text { different forest sample plots }(P<0.05) \text {. }\end{array}$} \\
\hline
\end{tabular}

\subsection{Structural characteristics of SMC}

Variation of soil PLFA biomarkers. 53, $62,69,61$ and 64 phospholipid fatty acids were detected in the soil samples of the sample plots in JMNR, and 38 PLFA biomarkers greater than $0.01 \mathrm{nmol} \cdot \mathrm{g}^{-1}$ were selected for further analysis (Table 4). The investigation of the spatial distribution of microorganisms by Zhou et al. (2015) showed significant differences in soil microorganisms in different locations. For this study, 
some PLFA biomarkers are completely distributed in five sample plots, such as 12:0 anteiso, 12:0, 13:0 anteiso, 14:0 anteiso, 15:0 anteiso, 16:1 $\omega 5 \mathrm{c}, 17: 1 \omega 7 \mathrm{c}$ 10-methyl, 18:1 $\omega 9 \mathrm{c}, 19: 0$ cyclo $\omega 7 \mathrm{c}$; some biomarkers are only distributed in specific single sample plot and are incomplete distribution, such as 11:0 anteiso, 14:0 iso, 15:1 $\omega 5$ c, 16:1 $\omega 9 c, 17: 1$ iso $\omega 9 c, 18: 1 \omega 7$ c 10-methyl, 20:0. 
Table 4

Content of PLFA biomarkers in different forest stands of Jinyun Mountain1 (nmol.g-1)

\begin{tabular}{|c|c|c|c|c|c|c|c|}
\hline No & $\begin{array}{l}\text { PLFA } \\
\text { biomarkers }\end{array}$ & Microorganisms & GA & CLF & PPF & PM\&GA & PM\&SS \\
\hline 1 & $11: 0$ anteiso & G+ & - & - & $\begin{array}{l}0.096 \pm \\
0.043 a b\end{array}$ & $\begin{array}{l}0.177 \pm \\
0.076 \mathrm{bc}\end{array}$ & $\begin{array}{l}0.175 \pm \\
0.079 c\end{array}$ \\
\hline 2 & G+ & G+ & $\begin{array}{l}0.144 \pm \\
0.078 a\end{array}$ & $\begin{array}{l}0.191 \pm \\
0.061 \mathrm{a}\end{array}$ & $\begin{array}{l}0.178 \pm \\
0.044 a\end{array}$ & $\begin{array}{l}0.243 \pm \\
0.080 a\end{array}$ & $\begin{array}{l}0.201 \pm \\
0.091 \mathrm{a}\end{array}$ \\
\hline 3 & $12: 0$ & Bacteria & $\begin{array}{l}0.122 \pm \\
0.048 a\end{array}$ & $\begin{array}{l}0.130 \pm \\
0.024 a\end{array}$ & $\begin{array}{l}0.109 \pm \\
0.039 a\end{array}$ & $\begin{array}{l}0.166 \pm \\
0.071 \mathrm{a}\end{array}$ & $\begin{array}{l}0.211 \pm \\
0.049 a\end{array}$ \\
\hline 4 & 13:0 anteiso & $\mathrm{G}+$ & $\begin{array}{l}0.154 \pm \\
0.028 a\end{array}$ & $\begin{array}{l}0.158 \pm \\
0.042 \mathrm{a}\end{array}$ & $\begin{array}{l}0.200 \pm \\
0.040 \mathrm{a}\end{array}$ & $\begin{array}{l}0.207 \pm \\
0.050 a\end{array}$ & $\begin{array}{l}0.182 \pm \\
0.045 a\end{array}$ \\
\hline 5 & $14: 0$ iso & G+ & $\begin{array}{l}0.047 \pm \\
0.006 a b\end{array}$ & $\begin{array}{l}0.076 \pm \\
0.027 \mathrm{~b}\end{array}$ & - & - & $\begin{array}{l}0.086 \pm \\
0.046 \mathrm{~b}\end{array}$ \\
\hline 6 & 14:0 anteiso & G+ & $\begin{array}{l}0.178 \pm \\
0.017 a\end{array}$ & $\begin{array}{l}0.155 \pm \\
0.058 a\end{array}$ & $\begin{array}{l}0.237 \pm \\
0.054 a\end{array}$ & $\begin{array}{l}0.200 \pm \\
0.021 \mathrm{a}\end{array}$ & $\begin{array}{l}0.196 \pm \\
0.023 a\end{array}$ \\
\hline 7 & 14:0 & Bacteria & $\begin{array}{l}0.230 \pm \\
0.067 \mathrm{a}\end{array}$ & $\begin{array}{l}0.343 \pm \\
0.156 a\end{array}$ & $\begin{array}{l}0.252 \pm \\
0.090 \mathrm{a}\end{array}$ & $\begin{array}{l}0.317 \pm \\
0.170 a\end{array}$ & $\begin{array}{l}0.472 \pm \\
0.291 \mathrm{a}\end{array}$ \\
\hline 8 & $15: 0$ iso & G+ & $\begin{array}{l}0.973 \pm \\
0.293 a\end{array}$ & $\begin{array}{l}1.177 \pm \\
0.720 \mathrm{a}\end{array}$ & $\begin{array}{l}1.022 \pm \\
1.005 a\end{array}$ & $\begin{array}{l}1.322 \pm \\
0.709 a\end{array}$ & $\begin{array}{l}1.563 \pm \\
0.776 a\end{array}$ \\
\hline 9 & $15: 0$ anteiso & G+ & $\begin{array}{l}0.497 \pm \\
0.111 \mathrm{a}\end{array}$ & $\begin{array}{l}0.531 \pm \\
0.199 a\end{array}$ & $\begin{array}{l}0.469 \pm \\
0.338 a\end{array}$ & $\begin{array}{l}0.568 \pm \\
0.224 a\end{array}$ & $\begin{array}{l}0.646 \pm \\
0.205 \mathrm{a}\end{array}$ \\
\hline 10 & $15: 1 \omega 7 c$ & G- & $\begin{array}{l}0.119 \pm \\
0.018 a\end{array}$ & $\begin{array}{l}0.118 \pm \\
0.021 \mathrm{a}\end{array}$ & $\begin{array}{l}0.119 \pm \\
0.021 \mathrm{a}\end{array}$ & $\begin{array}{l}0.107 \pm \\
0.024 a\end{array}$ & $\begin{array}{l}0.121 \pm \\
0.023 a\end{array}$ \\
\hline 11 & $15: 1 \omega 5 c$ & G- & $\begin{array}{l}0.649 \pm \\
0.022 \mathrm{~b}\end{array}$ & $\begin{array}{l}0.336 \pm \\
0.300 \mathrm{a}\end{array}$ & - & $\begin{array}{l}0.666 \pm \\
0.041 \mathrm{~b}\end{array}$ & $\begin{array}{l}0.699 \pm \\
0.075 \mathrm{~b}\end{array}$ \\
\hline 12 & 15:0 & Bacteria & $\begin{array}{l}0.130 \pm \\
0.033 a\end{array}$ & $\begin{array}{l}0.156 \pm \\
0.093 a\end{array}$ & $\begin{array}{l}0.131 \pm \\
0.064 \mathrm{a}\end{array}$ & $\begin{array}{l}0.182 \pm \\
0.109 a\end{array}$ & $\begin{array}{l}0.213 \pm \\
0.094 a\end{array}$ \\
\hline 13 & 15:0 DMA & $\begin{array}{l}\text { Anaerobic } \\
\text { bacteria }\end{array}$ & $\begin{array}{l}0.117 \pm \\
0.042 \mathrm{a}\end{array}$ & $\begin{array}{l}0.146 \pm \\
0.048 a\end{array}$ & $\begin{array}{l}0.172 \pm \\
0.084 a\end{array}$ & $\begin{array}{l}0.192 \pm \\
0.063 a\end{array}$ & $\begin{array}{l}0.221 \pm \\
0.089 a\end{array}$ \\
\hline 14 & $16: 3 \omega 6 c$ & Eukaryote & $\begin{array}{l}0.139 \pm \\
0.044 a\end{array}$ & $\begin{array}{l}0.128 \pm \\
0.042 \mathrm{a}\end{array}$ & $\begin{array}{l}0.087 \pm \\
0.033 a\end{array}$ & $\begin{array}{l}0.165 \pm \\
0.036 a\end{array}$ & $\begin{array}{l}0.141 \pm \\
0.034 a\end{array}$ \\
\hline 15 & $16: 0$ iso & G+ & $\begin{array}{l}0.583 \pm \\
0.199 a\end{array}$ & $\begin{array}{l}0.652 \pm \\
0.420 \mathrm{a}\end{array}$ & $\begin{array}{l}0.717 \pm \\
0.469 a\end{array}$ & $\begin{array}{l}0.945 \pm \\
0.676 a\end{array}$ & $\begin{array}{l}1.136 \pm \\
0.607 \mathrm{~b}\end{array}$ \\
\hline 16 & 16:0 anteiso & G+ & $\begin{array}{l}0.163 \pm \\
0.015 a\end{array}$ & $\begin{array}{l}0.173 \pm \\
0.019 a\end{array}$ & $\begin{array}{l}0.210 \pm \\
0.048 a\end{array}$ & $\begin{array}{l}0.175 \pm \\
0.012 a\end{array}$ & $\begin{array}{l}0.174 \pm \\
0.010 \mathrm{a}\end{array}$ \\
\hline 17 & $16: 1 \omega 9 c$ & G- & $\begin{array}{l}0.079 \pm \\
0.022 \mathrm{a}\end{array}$ & $\begin{array}{l}0.117 \pm \\
0.029 a\end{array}$ & - & $\begin{array}{l}0.084 \\
\pm .036 \mathrm{a}\end{array}$ & $\begin{array}{l}0.081 \pm \\
0.034 a\end{array}$ \\
\hline
\end{tabular}




\begin{tabular}{|c|c|c|c|c|c|c|c|}
\hline No & $\begin{array}{l}\text { PLFA } \\
\text { biomarkers }\end{array}$ & Microorganisms & GA & CLF & PPF & PM\&GA & PM\&SS \\
\hline 18 & $16: 1 \omega 7 c$ & G- & $\begin{array}{l}0.263 \pm \\
0.098 a\end{array}$ & $\begin{array}{l}0.397 \pm \\
0.238 a\end{array}$ & $\begin{array}{l}0.463 \pm \\
0.306 \mathrm{~b}\end{array}$ & $\begin{array}{l}0.358 \pm \\
0.198 a\end{array}$ & $\begin{array}{l}0.321 \pm \\
0.114 a\end{array}$ \\
\hline 19 & $16: 1 \omega 5 c$ & AM fungi & $\begin{array}{l}0.261 \pm \\
0.103 a\end{array}$ & $\begin{array}{l}0.295 \pm \\
0.155 b\end{array}$ & $\begin{array}{l}0.363 \pm \\
0.205 b\end{array}$ & $\begin{array}{l}0.293 \pm \\
0.174 a\end{array}$ & $\begin{array}{l}0.351 \pm \\
0.109 \mathrm{~b}\end{array}$ \\
\hline 20 & 16:0 & G- & $\begin{array}{l}3.131 \pm \\
0.691 \mathrm{a}\end{array}$ & $\begin{array}{l}3.711 \pm \\
1.463 \mathrm{~b}\end{array}$ & $\begin{array}{l}2.286 \pm \\
1.562 \mathrm{a}\end{array}$ & $\begin{array}{l}4.024 \pm \\
1.313 c\end{array}$ & $\begin{array}{l}4.571 \pm \\
0.940 c\end{array}$ \\
\hline 21 & $\begin{array}{l}\text { 16:0 10- } \\
\text { methyl }\end{array}$ & Actinomycetes & $\begin{array}{l}0.913 \pm \\
0.311 \mathrm{a}\end{array}$ & $\begin{array}{l}0.870 \pm \\
0.510 \mathrm{a}\end{array}$ & $\begin{array}{l}0.855 \pm \\
0.901 \mathrm{a}\end{array}$ & $\begin{array}{l}1.245 \pm \\
0.680 a\end{array}$ & $\begin{array}{l}1.601 \pm \\
0.589 a\end{array}$ \\
\hline 22 & $\begin{array}{l}17: 1 \text { iso } \\
\omega 9 \mathrm{c}\end{array}$ & G+ & - & $\begin{array}{l}1.102 \pm \\
0.120 \mathrm{~b}\end{array}$ & $\begin{array}{l}1.023 \pm \\
0.345 \mathrm{~b}\end{array}$ & - & - \\
\hline 23 & $17: 0$ iso & G+ & $\begin{array}{l}0.284 \pm \\
0.104 a\end{array}$ & $\begin{array}{l}0.379 \pm \\
0.227 \mathrm{a}\end{array}$ & $\begin{array}{l}0.291 \pm \\
0.254 a\end{array}$ & $\begin{array}{l}0.357 \pm \\
0.167 \mathrm{a}\end{array}$ & $\begin{array}{l}0.502 \pm \\
0.211 \mathrm{a}\end{array}$ \\
\hline 24 & 17:0 anteiso & G+ & $\begin{array}{l}0.346 \pm \\
0.053 a\end{array}$ & $\begin{array}{l}0.447 \pm \\
0.115 \mathrm{a}\end{array}$ & $\begin{array}{l}0.369 \pm \\
0.208 a\end{array}$ & $\begin{array}{l}0.392 \pm \\
0.082 \mathrm{a}\end{array}$ & $\begin{array}{l}0.574 \pm \\
0.275 a\end{array}$ \\
\hline 25 & $\begin{array}{l}\text { 17:0 cyclo } \\
\omega 7 \mathrm{c}\end{array}$ & G- & $\begin{array}{l}0.183 \pm \\
0.059 a\end{array}$ & $\begin{array}{l}0.269 \pm \\
0.161 \mathrm{a}\end{array}$ & $\begin{array}{l}0.254 \pm \\
0.155 a\end{array}$ & $\begin{array}{l}0.287 \pm \\
0.129 a\end{array}$ & $\begin{array}{l}0.353 \pm \\
0.118 a\end{array}$ \\
\hline 26 & $17: 0$ & Bacteria & $\begin{array}{l}0.094 \pm \\
0.038 \mathrm{~b}\end{array}$ & $\begin{array}{l}0.111 \pm \\
0.064 a b\end{array}$ & - & $\begin{array}{l}0.121 \pm \\
0.052 \mathrm{ab}\end{array}$ & $\begin{array}{l}0.145 \pm \\
0.042 \mathrm{~b}\end{array}$ \\
\hline 27 & $\begin{array}{l}\text { 17:1 w7c } \\
\text { 10-methyl }\end{array}$ & Actinomycetes & $\begin{array}{l}0.224 \pm \\
0.064 a\end{array}$ & $\begin{array}{l}0.309 \pm \\
0.112 \mathrm{~b}\end{array}$ & $\begin{array}{l}0.267 \pm \\
0.164 a\end{array}$ & $\begin{array}{l}0.275 \pm \\
0.108 a\end{array}$ & $\begin{array}{l}0.373 \pm \\
0.166 \mathrm{~b}\end{array}$ \\
\hline 28 & $\begin{array}{l}\text { 17:0 10- } \\
\text { methyl }\end{array}$ & Actinomycetes & $\begin{array}{l}0.181 \pm \\
0.083 a\end{array}$ & $\begin{array}{l}0.200 \pm \\
0.084 a\end{array}$ & $\begin{array}{l}0.172 \pm \\
0.118 \mathrm{a}\end{array}$ & $\begin{array}{l}0.295 \pm \\
0.128 \mathrm{a}\end{array}$ & $\begin{array}{l}0.243 \pm \\
0.103 a\end{array}$ \\
\hline 29 & $18: 3 \omega 6 c$ & G- & $\begin{array}{l}0.198 \pm \\
0.036 \mathrm{a}\end{array}$ & $\begin{array}{l}0.274 \pm \\
0.077 \mathrm{a}\end{array}$ & $\begin{array}{l}0.192 \pm \\
0.055 a\end{array}$ & $\begin{array}{l}0.265 \pm \\
0.083 a\end{array}$ & $\begin{array}{l}0.214 \pm \\
0.038 a\end{array}$ \\
\hline 30 & $18: 2 \omega 6 c$ & Fungi & $\begin{array}{l}0.392 \pm \\
0.074 a\end{array}$ & $\begin{array}{l}0.477 \pm \\
0.193 a\end{array}$ & $\begin{array}{l}0.279 \pm \\
0.119 a\end{array}$ & $\begin{array}{l}0.449 \pm \\
0.217 \mathrm{a}\end{array}$ & $\begin{array}{l}0.513 \pm \\
0.097 a\end{array}$ \\
\hline 31 & $18: 1 \omega 9 c$ & Fungi & $\begin{array}{l}0.826 \pm \\
0.343 a\end{array}$ & $\begin{array}{l}0.897 \pm \\
0.560 \mathrm{a}\end{array}$ & $\begin{array}{l}0.830 \pm \\
0.490 \mathrm{a}\end{array}$ & $\begin{array}{l}1.017 \pm \\
0.557 a\end{array}$ & $\begin{array}{l}1.052 \pm \\
0.281 \mathrm{a}\end{array}$ \\
\hline 32 & $18: 1 \omega 7 c$ & G- & $\begin{array}{l}0.469 \pm \\
0.204 a\end{array}$ & $\begin{array}{l}0.757 \pm \\
0.442 \mathrm{~b}\end{array}$ & $\begin{array}{l}0.512 \pm \\
0.283 a\end{array}$ & $\begin{array}{l}0.624 \pm \\
0.356 a\end{array}$ & $\begin{array}{l}0.505 \pm \\
0.250 \mathrm{a}\end{array}$ \\
\hline 33 & 18:0 & Bacteria & $\begin{array}{l}2.113 \pm \\
0.411 \mathrm{a}\end{array}$ & $\begin{array}{l}2.317 \pm \\
0.453 a\end{array}$ & $\begin{array}{l}1.727 \pm \\
0.616 \mathrm{a}\end{array}$ & $\begin{array}{l}2.397 \pm \\
0.311 \mathrm{a}\end{array}$ & $\begin{array}{l}2.776 \pm \\
0.298 a\end{array}$ \\
\hline 34 & $\begin{array}{l}18: 1 \omega 7 c \\
\text { 10-methyl }\end{array}$ & Actinomycetes & - & $\begin{array}{l}0.123 \pm \\
0.058 \mathrm{~b}\end{array}$ & - & $\begin{array}{l}0.138 \pm \\
0.043 b\end{array}$ & $\begin{array}{l}0.118 \pm \\
0.063 \mathrm{~b}\end{array}$ \\
\hline 35 & $\begin{array}{l}\text { 18:0 10- } \\
\text { methyl }\end{array}$ & Actinomycetes & $\begin{array}{l}0.177 \pm \\
0.083 a b\end{array}$ & $\begin{array}{l}0.280 \pm \\
0.144 c\end{array}$ & - & $\begin{array}{l}0.171 \pm \\
0.042 \mathrm{ab}\end{array}$ & $\begin{array}{l}0.259 \pm \\
0.118 \mathrm{c}\end{array}$ \\
\hline
\end{tabular}




\begin{tabular}{|c|c|c|c|c|c|c|c|}
\hline No & $\begin{array}{l}\text { PLFA } \\
\text { biomarkers }\end{array}$ & Microorganisms & GA & CLF & PPF & PM\&GA & PM\&SS \\
\hline 36 & $\begin{array}{l}\text { 19:0 cyclo } \\
\omega 7 \mathrm{c}\end{array}$ & G- & $\begin{array}{l}1.853 \pm \\
0.744 a\end{array}$ & $\begin{array}{l}1.815 \pm \\
1.016 a\end{array}$ & $\begin{array}{l}1.844 \pm \\
1.189 a\end{array}$ & $\begin{array}{l}2.169 \pm \\
1.099 a\end{array}$ & $\begin{array}{l}3.108 \pm \\
1.589 a\end{array}$ \\
\hline 37 & $20: 0$ & Bacteria & $\begin{array}{l}0.059 \pm \\
0.014 \mathrm{~b}\end{array}$ & - & - & $\begin{array}{l}0.080 \pm \\
0.022 b\end{array}$ & $\begin{array}{l}0.136 \pm \\
0.029 \mathrm{c}\end{array}$ \\
\hline 38 & $\begin{array}{l}\text { 20:0 10- } \\
\text { methyl }\end{array}$ & Actinomycetes & $\begin{array}{l}0.174 \pm \\
0.014 a\end{array}$ & $\begin{array}{l}0.295 \pm \\
0.155 a\end{array}$ & $\begin{array}{l}0.236 \pm \\
0.126 a\end{array}$ & $\begin{array}{l}0.339 \pm \\
0.092 a\end{array}$ & $\begin{array}{l}0.287 \pm \\
0.135 a\end{array}$ \\
\hline \multicolumn{3}{|c|}{$\begin{array}{l}\text { Total content of different PLFA } \\
\text { biomarkers }\end{array}$} & 16.464 & 20.010 & 15.963 & 21.013 & 24.518 \\
\hline \multicolumn{8}{|c|}{$\begin{array}{l}{ }^{1} \text { Different lowercase letters indicate the significance of the content differences of specific PLFA } \\
\text { biomarker for different forest sample plots }(P<0.05) \text {; "-" means that the PLFA biomarker is not } \\
\text { detected. }\end{array}$} \\
\hline
\end{tabular}

For the soils of the five forest sample plots, the most abundant PLFA biomarkers are 16:0 (indicator of Gbacteria), 18:0 (indicator of bacteria), 19:0 cyclo $\omega 7 \mathrm{c}$ (indicator of G-bacteria), $15: 0$ iso (indicator of G + bacteria) and 16:0 10-methyl (indicator of actinomycetes), indicating that their major roles in different soils of the five forest sample plots. Moreover, the contents of the four PLFA biomarkers 19:0 cyclo $\omega 7 \mathrm{c}$, 18:0, 15:0 iso, and 16:0 10-methyl are not significantly different in the sample plots, whose content of PM\&SS is the highest, followed by PM\&GA. The same situation applies to the PLFA biomarker of 16:0. Regarding the changes in phospholipid fatty acids, the contents of the five PLFA biomarkers (16:0, 18:0, 19:0 cyclo $\omega 7 \mathrm{c}, 15: 0$ iso and 16:0 10-methyl) in GA are $3.131,2.113,1.853,0.973$ and $0.913 \mathrm{nmol} \cdot \mathrm{g}^{-1}$, accounting for $54.56 \%$ of the total fatty acid content of GA. The contents of $16: 0,18: 0,19: 0$ cyclo $\omega 7 \mathrm{c}$, $15: 0$ iso and 16:0 10-methyl in CLF are $3.711,2.317,1.815,1.177$ and $0.870 \mathrm{nmol} \cdot \mathrm{g}^{-1}$, accounting for $49.42 \%$ of the total fatty acids in CLF, and their contents in PPF are 2.286, 1.727, 1.844, 1.022 and 0.855 $\mathrm{nmol} \cdot \mathrm{g}^{-1}$, accounting for $48.45 \%$ of the total fatty acids in PPF. The contents of $16: 0,18: 0,19: 0$ cyclo $\omega 7 \mathrm{c}$, 15:0 iso and 16:0 10-methyl in PM\&GA and PM\&SS are relatively high: their contents in PM\&GA are 4.024, $2.397,2.169,1.322$ and $1.245 \mathrm{nmol} \cdot \mathrm{g}^{-1}$, accounting for $55.54 \%$ of its total fatty acids; while their contents in PM\&SS are $4.571,2.776,3.108,1.563$ and $1.601 \mathrm{nmol} \cdot \mathrm{g}^{-1}$, accounting for $52.45 \%$ of its total fatty acids. Moreover, the order of the total PLFA biomarkers of the five forest stands is as follows: PM\&SS > PM\&GA > CLF > PM\&GA > PPF. In short, different forest stands have similar types of fatty acid with high content, but the distribution of PLFA biomarker content in each plot shows that the soil microbial PLFAs have certain spatial distribution characteristics for the forest stands in JMNR.

PCA of SMC structure. According to the PCA of SMC in different forest stand sample plots, the cumulative contribution rate of the two principal components related to the diversity of soil microbial PLFA community reached $68.62 \%$ (Fig. 2). Specifically, the first principal component (PC1) and the second principal component (PC2) explain $46.95 \%$ and $21.67 \%$ of the variance of the variables, respectively. Furthermore, 21 microbial PLFAs that play a significant role in PC1 have been identified, where 17:1 iso $\omega 9 \mathrm{c}$ and 20:0 10-methyl are positively correlated with PC1, while 20:0, 15:1 $\omega 5 c$, 11:0 
anteiso, 17:0, 18:0 10-methyl, 16:1 w9c, 16:3 w6c, 14:0 iso, 12:0 and 18:0 10-methyl are negatively correlated with PC1. The microbial PLFAs that play a significant role in PC2 are 16:0 anteiso, 15:0 anteiso, 15:1 $\omega 7 c, 18: 1 \omega 7 c, 16: 1 \omega 5 c, 13: 0$ anteiso and 18:3 $\omega 6 c$, all of which are positively correlated with PC2. The plots of the five forest stands are clearly distinguished. GA is mainly located at the negative end of PC1 and PC2, CLF is basically located at the positive end of PC1 and PC2, PPF is located at the positive end of PC1 and the positive and negative ends of PC2, and PM\&GA and PM\&SS are both located at the negative end of PC1 and the positive and negative ends of PC2. It is suggested that the main PLFAs affecting soil microorganisms in the five forest stands of JMNR are 17:1 iso $\omega 9 \mathrm{c}, 20: 0$ 10-methyl, 16:0 anteiso, 15:0 anteiso, 15:1 $\omega 7 c, 18: 1 \omega 7 c, 16: 1 \omega 5 c, 13: 0$ anteiso and 18:3 $\omega 6 c$.

a.11:0 anteiso,b.12:0 anteiso,c.12:0,d.13:0 anteiso,e.14:0 iso,f.14:0 anteiso,g.14:0,h.15:0 iso,i.15:0 anteiso,j.15:1 $\omega 7 \mathrm{c}, \mathrm{k} .15: 1 \omega 5 \mathrm{c}, \mathrm{l} .15: 0, \mathrm{~m} .15: 0$ DMA,n.16:3 $\omega 6 \mathrm{c}, 0.16: 0$ iso,p.16:0 anteiso,q.16:1 $\omega 9 \mathrm{c}, \mathrm{r} .16: 1$ $\omega 7 c, s .16: 1 \omega 5 c, t .16: 0, u .16: 0$ 10-methyl,v.17:1 iso $\omega 9 c, w .17: 0$ iso,x.17:0 anteiso,y. 17:0 cyclo $\omega 7 c, z .17: 0, A .17: 1 \omega 7 c$ 10-methyl,B.17:0 10-methyl,C.18:3 $\omega 6 c, D .18: 2 \omega 6 c, E .18: 1 \omega 9 c, F .18: 1$ $\omega 7 c, G .18: 0, H .18: 1 \omega 7 c$ 10-methyl,I.18:0 10-methyl,J.19:0 cyclo $\omega 7$ c,K.20:0,L.20:0 10-methyl; Numbers 1 $\sim 4$ represent the four sampling points of each of the five forest stands.

\subsection{Distribution characteristics of soil microorganisms}

As far as GA is concerned, the contents of each microbial group PLFA are ordered as $\mathrm{G}->\mathrm{G}+>$ actinomycetes > fungi > AM fungi, and for CLF, PPF, PM\&GA and PM\&SS, the order is: G + > G$>$ actinomycetes $>$ fungi $>$ AM fungi (Table 5). Furthermore, G-, G+, actinomycetes and fungi have the highest content in PM\&SS, while AM fungi have the highest content in PPF. Specifically, the five forest stands are ordered by the AM fungi as PPF $>$ PM\&SS forest $>C L F>P M \& G A>G A$, by $G+$ as PM\&SS forest $>\mathrm{CLF}>\mathrm{PPF}>\mathrm{PM} \& \mathrm{GA}>\mathrm{GA}$, by G- as $\mathrm{PM} \& \mathrm{SS}$ forest $>\mathrm{PM} \& \mathrm{GA}>\mathrm{GA}>\mathrm{CLF}>\mathrm{PPF}$, and by fungi (or actinomycetes) as PM\&SS $>$ PM\&GA forest $>$ CLF $>$ GA $>$ PPF. In addition, the order of G+/G- ratio in each forest stand is CLF $>$ PPF $>P M \& G A>P M \& S S>G A$, and the order of $F / B$ ratio is PPF $>G A>P M \& S S>C L F>$ $P M \& G A$. Altitude differences lead to differences in temperature and canopy closure of the five forest stands, and differences in tree age and latitude and longitude also lead to uneven solar radiation reaching specific sample plots. Therefore, it is suggested that the differences in soil microbial caloric conditions, natural geographical location and forest stand composition make the soil microbes show significant spatial distribution characteristics. 
Table 5

PLFA in G+, G-, Fungi, and Actinomycete and their ratio in different sample plots(nmol.g-1)

\begin{tabular}{|llllll|}
\hline $\begin{array}{l}\text { Microbial group } \\
\text { PLFA }\end{array}$ & Plots & & & \\
& GA & CLF & PPF & PM\&GA & PM\&SS \\
\hline AM fungi & $0.261 \pm$ & $0.295 \pm 0.173$ & $0.363 \pm 0.229$ & $0.293 \pm$ & $0.351 \pm$ \\
& 0.1035 & & & 0.1945 & 0.1085 \\
\hline G+ & $3.369 \pm 0.79$ & $5.0395 \pm$ & $4.8115 \pm$ & $4.5865 \pm$ & $5.448 \pm 2.31$ \\
& & 2.0365 & 2.6505 & 2.2155 & \\
\hline G- & $3.615 \pm$ & $3.4725 \pm$ & $3.1915 \pm$ & $4.2965 \pm 2.05$ & $5.16 \pm 2.146$ \\
& 1.0845 & 2.107 & 1.7795 & & \\
G+/G- & $0.956 \pm$ & $1.703 \pm 0.51$ & $1.422 \pm 0.188$ & $1.087 \pm 0.167$ & $1.051 \pm$ \\
& 0.145 & & & & 0.113 \\
\hline Fungi & $1.478 \pm$ & $1.6695 \pm$ & $1.473 \pm$ & $1.759 \pm$ & $1.916 \pm$ \\
\hline Fungi / bacteria & $0.111 \pm$ & $0.101 \pm 0.019$ & $0.126 \pm 0.029$ & $0.099 \pm 0.026$ & $0.102 \pm$ \\
& 0.019 & & & & 0.012 \\
\hline Actinomycetes & $1.6685 \pm$ & $2.1775 \pm$ & $1.5295 \pm 1.43$ & $2.4635 \pm$ & $2.881 \pm$ \\
& 0.504 & 0.8175 & & 1.518 & 0.913 \\
\hline
\end{tabular}

\subsection{Correlation between SMC diversity and soil factors}

RDA is used to analyze the correlation between SMC diversity, soil physical and chemical properties, and root system characteristics (Fig. 3). The two ranking axes explain a total of $95.82 \%$ of the SMC structure differences, of which the first axis and the second axis explain $91.5 \%$ and $4.32 \%$ of the SMC structure differences, respectively. Figure 3 shows that $\mathrm{G}+$ and $\mathrm{AM}$ fungi are significantly positively correlated with SOC, Ald, Alo, and Fed, and actinomycetes and fungi are significantly positively correlated with SOC, CEC, TN, and Fed. The above four microbial groups were significantly negatively correlated with AP, Sand, and the proportion of fine roots $(0.2-1)$. Moreover, G- is significantly positively correlated with SOC, CEC, and $\mathrm{TN}$, and significantly negatively correlated with $\mathrm{pH}, \mathrm{RLD}$, the proportion of very fine roots $(0-0.2)$, and AP. The G+/G- ratio is significantly positively correlated with RLD, $\mathrm{pH}, \mathrm{AP}$ and the proportion of very fine roots, and significantly negatively correlated with $A D, T N, C E C, S O C$ and the proportion of fine roots. The ratio of F/B was significantly positively correlated with $T N$ and $A D$, and significantly negatively correlated with $A P$, $\mathrm{pH}$, and the proportion of very fine roots.

\section{Discussion}

\subsection{Distribution characteristics of SMC in different forest stands}


The content of PLFA biomarkers can reflect the total amount of soil microorganisms in five different forest stands. Therefore, soils under the five forest stands of GA-EBF, CLF, PPF, PM\&GA and PM\&SS were collected from JMNR, and 53, 62, 69, 61 and 64 PLFAs were detected from them by PLFA analysis, respectively. Compared with other forest ecosystems (Huang et al. 2007; Luo et al. 2017), the microbial PLFA species in JMNR soil are rich, but the PLFA content is relatively low. On the one hand, subtropical forest soil has rich vegetation characteristics and diverse types of available nutrients, resulting in a diversity of microbial species, which echoes the results of Zhou et al. (2017). On the other hand, due to long-term acid rain stress, the JMNR soil is slightly acidic, indicating that the acidic environment has a certain inhibitory effect on the microbial content. Furthermore, the research results show that the species with higher PLFA content in JMNR soil microorganisms are basically the same, mainly reflected in 16:0, 18:0, 19:0 cyclo $\omega 7 \mathrm{c}, 15: 0$ iso and 16:0 10-methyl. As described above, the soil of JMNR is basically acidic, and the above five PLFA types are the main biomarkers of bacteria, suggesting that the bacterial community in the JMNR ecosystem occupies a major position, which is different from other forests. This finding is different from the previous documents (Rousk et al. 2010; Narendrul 2003; Mühlbachová and Tlusto 2006), which found that fungi are more tolerant to acidic soils than bacteria, while is consistent with the findings of Li et al. (2012)and Jiang et al. (2013), who found that the PLFAs content of soil bacteria in different forest stands was significantly higher than that of fungi and actinomycetes. This phenomenon may be due to SMC's preference for environmental conditions such as water, air, and sunlight, and the differences in soil properties in different climatic regions and forest types (He and $\mathrm{Ge}$ 2008; Chaer et al. 2009). Specifically, the long-term acid rain climate in the JMNR area caused changes in the type and quantity of organic matter, which affected the soil nutrient environment, and the tolerance of bacteria to acid rain exceeds that of fungi, which eventually led to changes in the structure of SMC. Moreover, considering that the soil layer of JMNR is rich in nutrients (Liu 2019), in the process of soil nutrient cycling, bacterial pathways mainly occur in nutrient-rich soils that are rich in easily decomposable organic matter and have a faster carbon turnover and nutrient cycling rates (Zhou et al. 2017; Ingwersen et al. 2007).

The results showed that the structure and component content of SMC in different forest stands are significantly different. For the five forest stands, G-, G+, actinomycetes, and fungi all have the highest content in PM\&SS, while AM fungi have the highest content in PPF. In terms of total PLFA biomarkers, PM\&SS and PM\&GA are the highest, followed by CLF and GA, and PPF is the least. This may be attributed to the different accumulation and storage of SOC in different forest types (Zi et al. 2017). Specifically, the reasonable spatial distribution pattern of CBLMF can promote microbial activity and increase soil enzyme activity, thereby accelerating the decomposition of organic matter and nutrient accumulation, and improving soil's physical and chemical properties. Thus CBLMF is more conducive to the survival of microorganisms than other forests (Huang et al. 2007; Zhang et al. 2019). Furthermore, the amount of litter accumulation in CBLMF is more abundant than that of other forest stands. Guo et al. (2016) found that tree species significantly influence the SMC structure through research on the natural Chinese pine forest, CLF and artificial PM. Hong et al. (2016) obtained similar results through PCA of soil PLFA biomarkers in Castanopsis hystrix and Erythrophleum fordii. In addition, several other studies also 
support this finding, believing that the influence of tree species on the structure of SMC is mainly due to the indirect impact of the difference in the quality and quantity of the aboveground and underground inputs of the soil between the tree species on the soil properties (Masayuki et al. 20108; Chen et al. 2013). $\mathrm{Zi}$ et al. (2017) and Zheng et al. (2004) found that the soil microbial content of CBLMF is higher than other forest stands in their research on the Qinghai-Tibet Plateau and the red soil hilly area of Jiangxi, which is further confirmed by this study. The ratio of F/B in SMC can reflect the stability and resilience of the microbial community. This study found that the ratio of F/B in PPF is higher than other forest stands, and fungi in CBLMF have higher PLFAs, indicating that PM\&SS and PM\&GA have better microbial resistance, which is conducive to efficient resource utilization, while the SMC of PPF has lower resistance, but has better resilience. This phenomenon is due to the well-developed root system of PPF, and its surface is covered with more litter, which can maintain nutrient supply in the case of damage and provide facilities for later vegetation restoration. The spatial structure of different forest stands is disturbed by factors such as light, temperature, moisture, air, which also affect the carbon and nitrogen content of the soil. For example, the more litter in a forest stand, the content of actinomycetes will increase to better degrade complex carbon compounds and resist more severe abiotic stress in the soil, and the content of fungi will also change to better decompose organic matter that is difficult to decompose (Zhou 2005; Roes-Hill et al. 2011).

\subsection{Correlation between soil factors and SMC composition}

The influencing factors of soil formation are complex and diverse, especially for the subtropical forest ecosystem, which has a certain impact on the spatial distribution of SMC. By observing a number of soil property indicators, the main driving factors for changes in the SMC structure of different forest stands were determined. The results showed that for the five forest stands, there were significant differences in soil $\mathrm{pH}, \mathrm{AP}$ content, soil texture, and CEC, while the differences in SOC, TN, Fed, Ald, Feo, and Alo content were not significant. RDA analysis shows that only some of the above variables contribute to the change of SMC structure. Specifically, AM fungi, G+, G-, fungi, and actinomycetes are significantly positively correlated with Fed, Ald, Feo, Alo, SOC, TN, CEC and AD, and negatively correlated with AP, pH, the proportion of very fine roots, and sand content. The most important influencing factors are the content of iron and aluminium oxides, SOC and TN in the soil, which explain $52.5 \%$ of SMC's structural variation. Among them, the influence of SOC on the structure of SMC has been reported many times in previous studies. As a source of energy and material required for the life activities of soil microorganisms, the quality and quantity of SOC have an important impact on the activity and structure of microorganisms and can affect the utilization of organic carbon substrates by microorganisms. Tian et al. (Tian et al. 2012) found that the change of land use structure affects the type and quantity characteristics of SOC, and has a significant impact on the structure of SMC. Masayuki et al. (Masayuki et al. 20108) found that the abundance of specific microbial markers in coniferous forests and broad-leaved forests is related to the total amount of SOC. According to Asemaninejad et al. (Asemaninejad et al. 2016), changes in fungal communities are more dependent on soil carbon quality and composition, and SOC and TN are important factors that cause differences in PLFAs. Although soil pH is considered one of the driving factors of SMC structure (Tripathi et al. 2012; Krashevska et al. 2015), this study found that pH has no significant effect 
on SMC structure. This may be because the soil in the study area has been facing acid rain climate for a long time, leading to the adaptability of microorganisms to the acidic environment, and the effect of soil acidification on microorganisms is mainly reflected by changes in the nutrient environment such as organic matter and the ratio of carbon/nitrogen. Hu (Hu 2018) found that soil acidification caused by simulated acid rain changed soil properties, inhibited the effective use of carbon by microorganisms, disrupted the soil carbon and nitrogen cycle process, and affected the structure of SMC (Taina et al. 1998 ;Liu et al. 2017).

Prescott and Grayston (Prescott and Grayston 2013) found that, in addition to specific microorganisms related to certain trees, vegetation causes changes in the SMC structure through indirect mechanisms rather than direct ones. Furthermore, the root system of plants is also one of the factors in this indirect mechanism. Further analysis showed that most of the microbial PLFAs in the soil are significantly affected by the biomass of fine roots, especially the RLD and the proportion of fine roots. Plant roots have strong physiological activity and metabolism, e.g., dead roots can provide organic matter, thereby improving soil's physical and chemical properties (Zhan 2017). In this study, the significant effect of fine roots may be due to their strong absorption capacity for nutrients and water. The proportion of fine roots in different forest stands is different, resulting in a certain difference in SMC. Moreover, Luo et al. (Luo et al. 2017) found that different forest types and plant compositions caused changes in the amount of litter and its decomposition rate, affecting the quality and quantity of nutrients in the soil and the composition and SMC's diversity.

\section{Conclusions}

For the understory soil of JMNR, the microbial PLFAs have a high diversity. Specifically, for the five forest stands, 53, 62, 69, 61 and 64 PLFAs were detected. Among them, 16:0, 19:0 cyclo $\omega 7 \mathrm{c}, 18: 0,15: 0$ iso, and 16:0 10-methyl are the dominant PLFAs in the soil, and 16:0 (G- bacteria) is the largest content for all of the five forest stands.

In terms of the number of soil microorganisms and their community structure, there are significant differences among the five forest stands of JMNR. The total content of PLFA biomarkers is the highest in the two CBLMFs of PM\&SS and PM\&GA, followed by CLF and GA, and PPF has the least amount. Moreover, the PLFA content of different microbial groups in GA is ranked as G$>$ G+>actinomycetes>fungi>AM fungi, while ranked as PM\&GA and PM\&SS, G+>G$>$ actinomycetes $>$ fungi>AM fungus in CLF, PPF, PM\&GA, and PM\&SS.

AM fungi, G+, G-, fungi, and actinomycetes are significantly positively correlated with Fed, Ald, Feo, Alo, SOC, TN, CEC and AD, and negatively correlated with $A P, \mathrm{pH}$, the proportion of very fine roots, and soil sand. Furthermore, the main influencing factors of PLFAs are the content of iron and aluminium oxides, SOC and TN in the soil, which can effectively reflect the nutrient status of the soil in JMNR.

Regarding the SMC structure of the five forest stands in JMNR, PPF has the highest soil microbial diversity but has no abundance advantage; the two CBLMFs of PM\&SS and PM\&GA have higher soil 
microbial diversity and abundance advantages.

\section{Declarations}

\section{Ethics approval and consent to participate}

Not applicable

\section{Consent for publication}

Not applicable

\section{Availability of data and material}

Not applicable

\section{Competing interests}

The authors declare that they have no competing interests.

\section{Funding}

This work was supported by the National Key Research and Development Program of China (grant numbers 2017YFC0505303).

\section{Authors' contributions}

Y.W. planned and supervised the project, reviewed drafts of the paper. X.A, and J.Z. conceived, designed and performed the experiments. X.A. analyzed the data, made charts and led manuscript production. All authors have read and agreed to the published version of the manuscript.

\section{Acknowledgements}

We would like to thank Institute of Soil Scienc, Chinese Academy of Sciences for completing the phospholipid fatty acid analysis.

\section{References}

1. Abaye DA, Lawlor K, Hirsch PR, Brookes PC (2005) Change in the microbial community of an arable soil caused by long-term metal contamination. Eur J Soil Sci 56:93-102

2. Alexander K, Bryans $T$ (2006) Evaluation of the sterility test for detection of microbial contaminants of allografts. Cell Tissue Banking 7:23-28

3. Asemaninejad A, Thorn RG, Lindo Z (2017) Experimental climate change modifies edgradative succession in boreal peatland fungal communities. Microb Ecol 73:521-531 
4. Bao TL, Gao LQ, Wang SS, Yang XQ, Ren W, Zhao YG (2020) Moderate disturbance increases the PLFA diversity and biomass of the microbial community in biocrusts in the Loess Plateau region of China. Plant Soil 451:499-513

5. Barrow J, Kaur R, Al E (2005) Delivering Shared Heritage: The Mayor's commission on African and Asian heritage. Greater London Authority

6. Chen F, Zheng H, Zhang K, Ouyang Z, Lan J, Shi Q (2013) Changes in soil microbial community structure and metabolic activity following conversion from native Pinus massoniana plantations to exotic Eucalyptus plantations. For Ecol Manage 291:65-72

7. Chen ZL, Shu YG, Zhou PP (2020) Exchange and distribution characteristics of soil base ions under different ecological restoration pattern in the Karst mountainous area. J Soil Water Conserv 34:304319. (In Chinese)

8. Dangi S, Gao S, Duan YH, Wang D (2019) Soil microbial community structure affected by biochar and fertilizer sources. Appl Soil Ecol 150:103452

9. Dangi SR, Banuelos G, Buyer JS, Hanson B, Gerik J (2018) Microbial community biomass and structure in saline and non-saline soils associated with salt-and boron-tolerant poplar clones grown for the phytoremediation of selenium. Int J Phytorem 20:129-137

10. Dangi SR, Tirado-Corbala R, Cabrera JA, Wang D, Gerik J (2014) Soil biotic and abiotic responses to dimethyl disulfide spot drip fumigation in established grape vines. Soil Sci Soc Am J 78:520-530

11. Ding JJ, Zhang YG, Deng Y, Cong J, Lu H, Sun X, Yang CY, Yuan T, Van NJD, Li DQ, Zhou JZ, Yang YF (2015) Integrated metagenomics and network analysis of soil microbial community of the forest timberline. Sci Rep 5:1-10

12. Fierer N, Leff JW, Adams BJ, Nielsen UN, Bates ST, Lauber CL, Owens S, Gilbert JA, Wall DH, Caporaso JG (2012) Cross-biome metagenomics analyses of soil microbial communities and their functional attributes. Proceedings of the National Academy of Sciences USA, 109: 21390-21395

13. Frostegård A, Tunlid A, Bååth $E$ (1993) Phospholipid atty acid composition, biomass and activity of microbial communities from two soil types experimentally exposed to different heavy metals. Appl Environ Microbiol 59:3605-3617

14. Guilherme MC, Marcelo FF, David DM, Peter JB (2009) Shifts in microbial community composition and physiological profiles across a gradient of induced soil degradation. Soil Sci Soc Am J 73:13271334

15. Guo J, Yang Z, Lin C, Liu X, Chen G, Yang Y (2016) Conversion of a natural evergreen broadleaved forest into coniferous plantations in a subtropical area: effects on composition of soil microbial communities and soil respiration. Biology Fertility of Soilsbiol Fert Soils 52:799-809

16. He J, Ge Y (2008) Recent advances in soil microbial biogeography. Acta Ecol Sin 28:5571-5582. (In Chinese)

17. Herrick JE, Hefin J (2013) Soil ecology and ecosystem services. Oxford University Press, Oxford

18. Hong P, Liu S, Wang H, Yu H (2016) Characteristics of soil microbial community structure in two young plantations of Castanopsis hystrix and Erythrophleum fordii in subtropical China. Acta Ecol 
Sin 36:4496-4508. (In Chinese)

19. Hu B (2018) Dynamics of aggregate stability of the yellow soil under acid rain background in the Three Gorges Reservoir area. Beijing Forestry University, (In Chinese)

20. Hu B, Zhang H, Wang B, Wang Y, Guo P, Liu C, Tang X (2015) Analysis on the forest soil acidification and mechanisms in ChongQing Jinyun Mountain. Resources and Environment in the Yangtze Basin, 24: 300-309. (In Chinese)

21. Huang Z, Tian D, Liang R, Zhou G (2007) Quantitative characteristics of soil micro-organisms of different stands in Nanling Mountains,South China(In Chinese). Journal of Central South University of Forestry Technology 27:1-5

22. Ingwersen J, Poll C, Streck T, Kandeler E (2008) Micro-scale modelling of carbon turnover driven by microbial succession at a biogeochemical interface. Soil Biol Biochem 40:864-878

23. Jiang Y, Xiao J, Luo C, Li J, Zhang G (2014) The microbial community of three mountains Pingding,Changbai and Saihanwula based on the analysis of phospholipid fatty acids (PLFAs). Ecology Environmental Sciences 23:1272-1279. (In Chinese)

24. Krashevska V, Klarner B, Widyastuti R, Maraun M, Scheu S (2015) Impact of tropical lowland rainforest conversion into rubber and oil palm plantations on soil microbial communities. Biol Fertil Soils 51:697-705

25. Li J, Jiang C, Hao Q (2015) Distribution characteristics of soil organic carbon and its physical fractions under the different land uses in Jinyun Mountain. Acta Ecol Sin 35:3733-3742. (In Chinese)

26. Li Y, Gu Y, Liu Y, Huang J, Yang H (2012) Characteristics of microbial quantities and communities in forest soils of Jinyun Mountain. Plant Nutrition Fertilizer Science 18:1161-1167. (In Chinese)

27. Liu C (2019) Effects of structure characteristics of typical forest stands on slope hydrological processes in Jinyun Mountain, Chongqing. Beijing Forestry University, (In Chinese)

28. Liu X, Zhang B, Zhao W, Wang L, Xie D, Huo W, Wu Y, Zhang J (2017) Comparative effects of sulfuric and nitric acid rain on litter decomposition and soil microbial community in subtropical plantation of Yangtze River Delta region. Sci Total Environ 601:669-678

29. Luo D, Liu S, Shi Z, Feng Q, Liu Q, Zhang L, Huang Q, He J (2017) Soil microbial community structure in Picea asperata plantations with different ages in subalpine of western Sichuan,Southwest China. Chin J Appl Ecol 28:519-527. (In Chinese)

30. Mühlbachová G, Tlustoš $P$ (2006) Effects of liming on the microbial biomass and its activities in soils long-term contaminated by toxic elements. Plant Soil Environment 52:345-352

31. Narendrula R, Nkongolo KK (2015) Fatty acids profile of microbial populations in a miningreclaimed regioncontaminated with metals:relation with ecological characteristics and soil respiration. Environ Model Softw 6:1-9

32. Ou W, Li Q, Liang WJ, Jiang Y, Wen DZ (2004) Effects of water regimes on soil biological properties in a paddy field. Chin J Ecol 23:53-56 
33. Peay KG, Kennedy PG, Bruns TD (2011) Rethinking ectomycorrhizal succession: are root density and hyphal exploration types drivers of spatial and temporal zonation. Fungal Ecology 4:233-240

34. Pennanen T, Perkiömäki J, Kiikkilä O, Pekka V, Seppo N, Hannu F (1998) Prolonged, simulated acid rain and heavy metal deposition: separated and combined effects on forest soil microbial community structure. FEMS Microbiol Ecol 27:291-300

35. Prescott CE, Grayston SJ (2013) Tree species influence on microbial communities in litter and soil: current knowledge and research needs. For Ecol Manage 309:19-27

36. Roes-Hill M, Khan N, Burton SG (2011) Actinobacterial peroxidases: an unexplored resource for biocatalysis. Appl Biochem Biotechnol 164:681-713

37. Rousk J, Bååth E, Brookes PC, Lauber CL, Lozupone C, Caporaso JG, Knoght R, Fierer N (2010) Soil bacterial and fungal communities across a pH gradient in anarable soil. ISME J 4:1340-1351

38. Shen CC, Xiong JB, Zhang HY, Feng YZ, Lin XG, Li XY, Liang WJ, Chu HY (2013) Soil pH drives the spatial distribution of bacterial communities along elevation on Changbai mountain. Soil Biol Biochem 57:204-211

39. Tian J, Fan M, Guo J, Marschner P, Li X, Kuzyakov Y (2012) Effects of land use intensity on dissolved organic carbon properties and microbial community structure. European Journal of Soil Biology $52: 67-72$

40. Tripathi BM, Kim M, Singh D, Lee-Cruz L, Lai-Hoe A, Ainuddin AN (2012) Tropical soil bacterial communities in Malaysia: pH dominates in the equatorial tropics too. Microb Ecol 64:474-484

41. Ushio M, Wagai R, Balser TC, Kitayama K (2008) Variations in the soil microbial community composition of a tropical montane forest ecosystem: does tree species matter. Soil Biol Biochem 40:2699-2702

42. Wang S, Xu JP, Wang N, Lei WY, Fan XY, Dou S (2018) Structural characteristics of mineral-microbial residues formed by microbial utilization of Lignin Joined with Fe, Al, Mn-Oxides based on FT-IR and SEM techniques. Spectroscopy Spectral Analysis 38:2086-2093. (In Chinese)

43. Wang YT, Wang ZF, Huang R, Lü S, Gao M (2019) Characterization of soil microbial community structure as affected by vegetation in Jinyun Mountain. Acta Pedol Sin 5:1210-1220. (In Chinese)

44. Weil R, Brady N (2017) The Nature and Properties of Soils. 15th edition

45. Xiang ZY, Zhang L, Zhang QF, Liu W, Wang GX, Wang CT, Hu L (2014) Soil nutrients and microbial functional diversity of different stand types in Qinghai rovince. Scientia Silvae Sinicae 50:22-31. (In Chinese)

46. Xu ZJ, Wan XH, Liang YF, Shi XZ (2021) Effects of root growth on leaf litter decomposition and enzyme activity in litter layer. Chin J Appl Ecol 32:31-38. (In Chinese)

47. Zeng Q, He B (2016) Effect of nitrogen deposition on soil microbial community structure determined with the PLFA method under the Masson pine forest from Mt. Jinyun Chongqing Environmental Science 37:3590-3597. (In Chinese) 
48. Zhan H (2017) Effects of Root Characteristics of Three Herbaceous Plants on Soil Erodibility. Huazhong Agricultural University, (In Chinese)

49. Zhang $Y$, Fang $X$, Xian $Y$, Wang Z, Xiang W (2019) Characteristics of soil microbial biomass carbon, nitrogen, phosphorus and enzyme activity in four subtropical forests. China Acta Ecologica Sinica 39:5326-5338. (In Chinese)

50. Zheng S, Wu W, He Y (2004) Microbial groups and enzyme activity under different artificial forestry in hilly red soil. Jiangxi Forestry Science Technology 4:1-4. (In Chinese)

51. Zhou D (2005) Litter of mixed forest of Cunninghamia lanceolata and Manglietia yuyuanensis (In Chinese). Chin J Ecol 24:595-598

52. Zhou S, Liang YT, Sun B (2015) Research Advances in Environmental and Human Factors Affecting Microbial Community Structure and Evolution in Red Soils[J]. Soils 7:272-277

53. Zhou Y, Qiao S, Liu J, Jia T, Li C, Chai B, Zhang N, Liang Z (2017) Characteristics of the soil microbial community structures of Betula platyphylla and Larix principis-rupprechtii forests in the Pangquangou Reserve. Chinese Journal of Applied Environmental Biology 23:520-526. (In Chinese)

54. Zi H, Xiang Z, Wang G, Ade L, Wang C (2017) Profile of microbial community under different stand types in Qinghai Province. Scientia Silvae Sinicae 53:21-32. (In Chinese)

55. Zuo YL, He XL, Wang SJ, Zhao LL (2016) Characteristics of soil microbial community structure in the Rhizospheric soil of Ammopoptanthus mongolicus by phospholipid fatty acid. Environmental Science 37:2705-2713. (In Chinese)

\section{Figures}

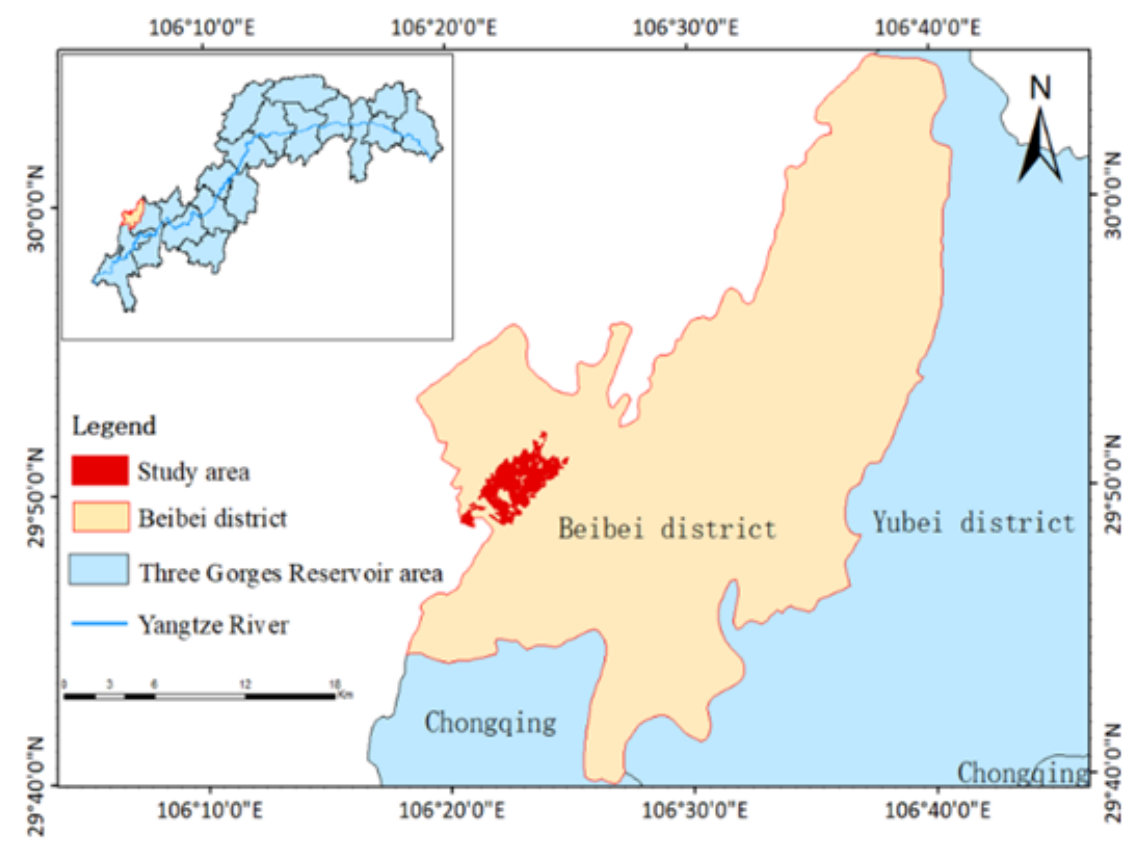



PPF

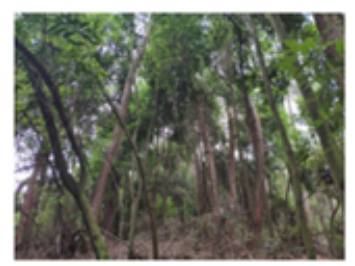

CBLMF of PM \&SS
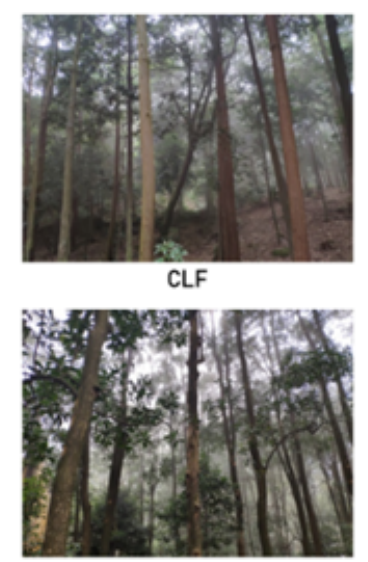

CBLMF of PM\&GA

\section{Figure 1}


Geographical location of the study area.

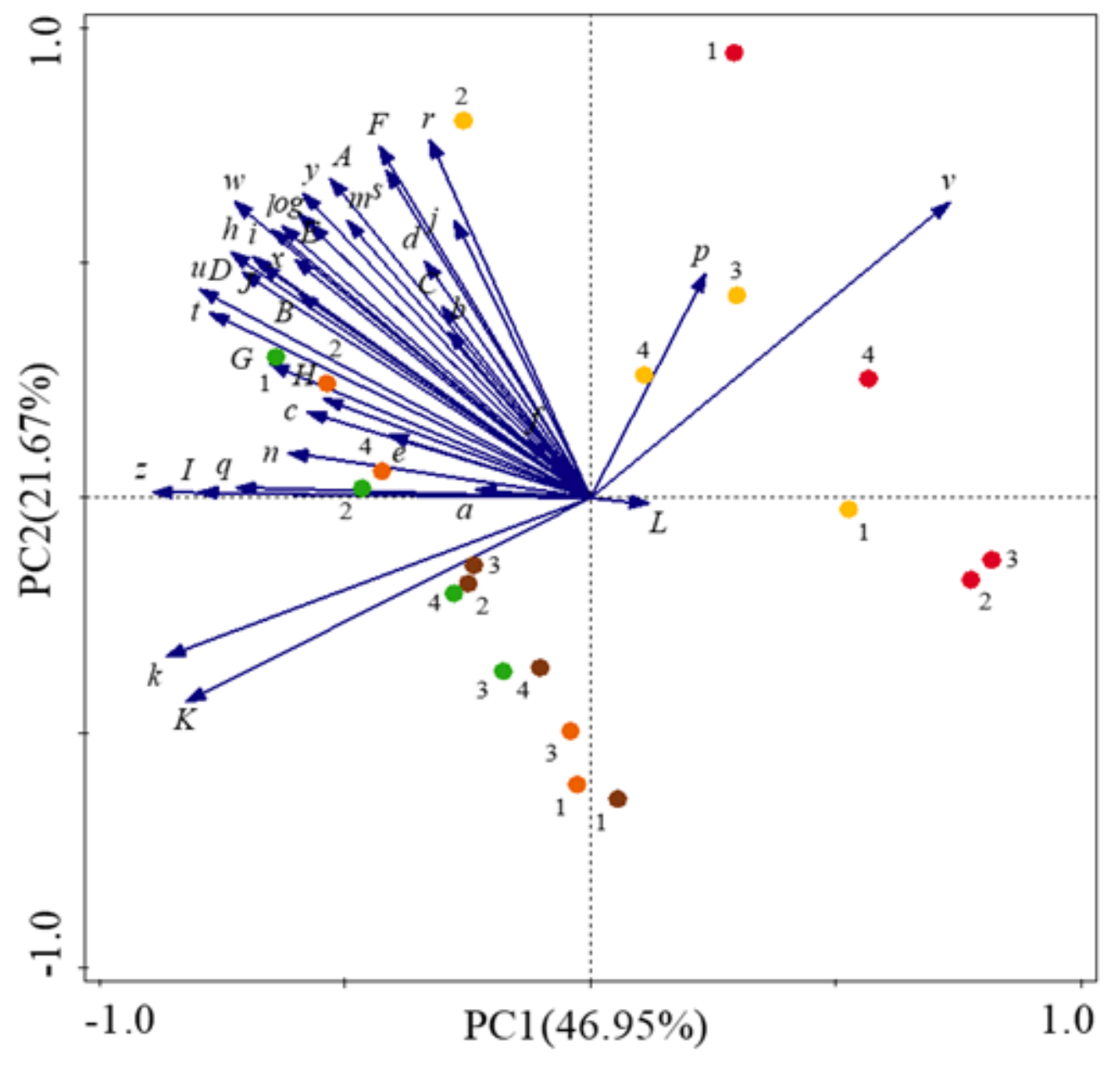

- GA-EBF

CLF

- PPF

CBLMF of PM\& GA

- CBLMF of PM\&SS

\section{Figure 2}

PCA of different microbial group PLFA contents in different sample plots. a.11:0 anteiso,b.12:0 anteiso,c.12:0,d.13:0 anteiso,e.14:0 iso,f.14:0 anteiso,g.14:0,h.15:0 iso,i.15:0 anteiso,j.15:1 $\omega 7$ c,k.15:1 $\omega 5$ c,l.15:0,m.15:0 DMA,n.16:3 $\omega 6$ c,o.16:0 iso,p.16:0 anteiso,q.16:1 $\omega 9$ c,r.16:1 $\omega 7$ c,s.16:1 $\omega 5 c, t .16: 0, u .16: 0$ 10-methyl,v.17:1 iso $\omega 9 c, w .17: 0$ iso,x.17:0 anteiso,y. 17:0 cyclo $\omega 7 c, z .17: 0, A .17: 1 \omega 7 c$

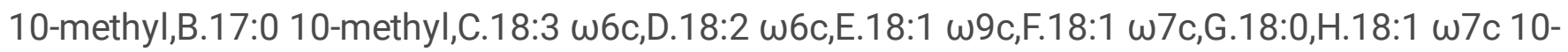
methyl,I.18:0 10-methyl,J.19:0 cyclo $\omega 7$ c,K.20:0,L.20:0 10-methyl; Numbers 1 4 represent the four sampling points of each of the five forest stands. 


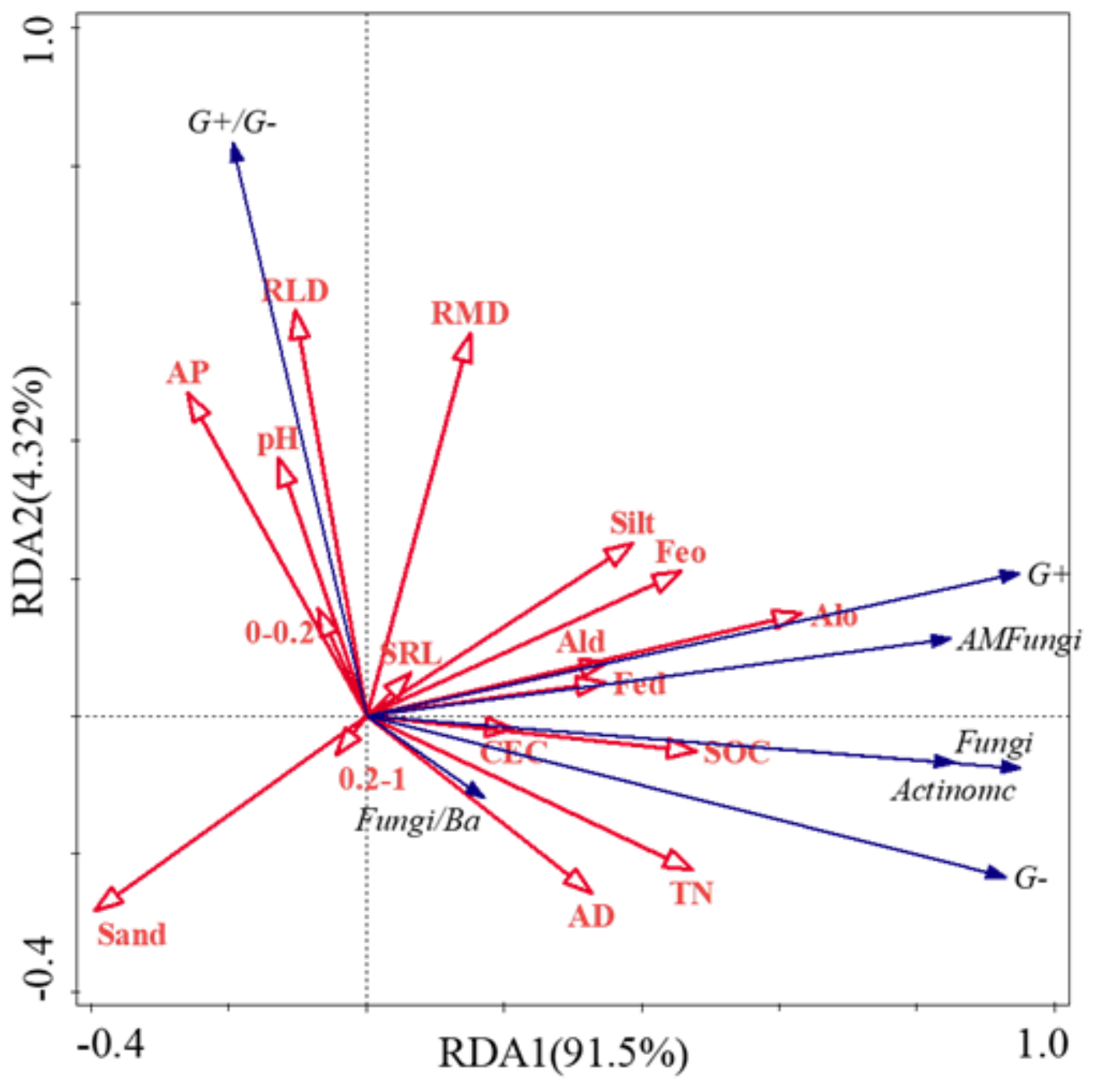

Figure 3

RDA results of soil microbial community and soil factors. 